\title{
Small bowel haemangioma with local lymph node involvement presenting as intussusception
}

\author{
D R Morgan, K Mylankal, N El Barghouti, M F Dixon
}

\begin{abstract}
Gastrointestinal haemangiomas make up $0.05 \%$ of all intestinal neoplasms. They are sometimes multiple and usually present with pain, bleeding, and obstruction. An associated haemangiomatous change in regional lymph nodes has not been reported previously. A woman of 21 years presented with abdominal pain and vomiting. Abdominal ultrasound and computed tomography scan showed a lower abdominal mass. Laparotomy revealed a small bowel tumour causing an intussusception together with enlarged mesenteric lymph nodes. Pathological examination revealed a small bowel haemangioma with mesenteric node involvement. The pathogenesis of haemangiomatous involvement of lymph nodes is discussed. Hamartomatous change is the likely cause in this patient.

(F Clin Pathol 2000;53:552-553)
\end{abstract}

Keywords: haemangioma; lymph node; intussusception; small bowel

Haemangiomas of the gastrointestinal tract are uncommon, accounting for only $0.05 \%$ of all intestinal neoplasms. ${ }^{1}$ River et al collected 127 cases of small bowel haemangiomas that were evenly distributed throughout the jejunum and ileum. ${ }^{2}$ They have a tendency towards multiplicity and an association with systemic angiomatoses such as blue rubber bleb naevus syndrome, Maffucci's syndrome, and KlippelTrenaunay-Weber syndrome. ${ }^{34}$ Bleeding, chronic anaemia, and abdominal pain are the most common presenting features, with obstruction and intussusception being reported. ${ }^{5}$ Malignant change ${ }^{6}$ is very rare, but has been documented, as has an association with protein losing enteropathy ${ }^{7}$ and mucocutaneous pigmentation. ${ }^{8}$ We report a case of small bowel haemangioma presenting as intussusception in association with a highly unusual benign haemangiomatous change in the regional lymph nodes, and explore the possible pathogenesis of this.

Surgery, Scarborough Hospital

K Mylankal

N El Barghouti

Academic Unit of Pathology, University of Leeds, Leeds LS2 9JT, UK

M F Dixon

Correspondence to: Dr Morgan

email: morganr@

mail.scarney.northy.nhs.uk

Accepted for publication 7 January 2000 but apyrexial with abdominal tenderness in the peri-umbilical region. There were no signs of peritonism. Bowel sounds were absent and

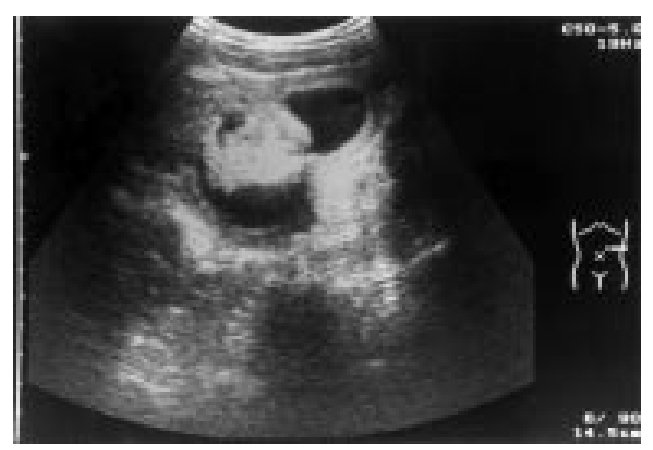

Figure 1 Ultrasound results. Transverse image of left mid abdomen showing a $5 \mathrm{~cm}$ in diameter intraluminal lobulated tumour within the dilated small bowel.

digital examination of the rectum was unremarkable. An ultrasound of the abdomen showed a large lobulated mass with multilayered appearance, suggestive of an intussusception with evidence of associated small bowel obstruction (fig 1). Gastrograffin enema revealed a normal large bowel. A computed tomography (CT) scan of the abdomen confirmed the presence of a laminated mass in the lower abdomen. She was initially managed conservatively with intravenous fluids and nasogastric aspiration. Her symptoms failed to improve with these conservative measures and consequently she underwent a laparotomy, which revealed a mid small bowel intussusception caused by a tumour, together with enlarged lymph nodes in the mesentery. The affected loop of small bowel was resected en mass with the lymph nodes and an end to end anastomosis was performed. The postoperative period was uneventful and she was discharged home on the fourth day.

Gross pathological examination revealed a polypoid small bowel tumour measuring $4 \times 4 \times 5 \mathrm{~cm}$ arising from the submucosa together with eight lymph nodes, the largest $1.1 \mathrm{~cm}$ in diameter, in the mesentery. Microscopy revealed the small bowel lesion to be composed of multiple small and large blood filled channels lined by plump endothelial cells with scattered mitoses but no pronounced cytological atypia or other features of malignancy (fig 2). These changes were predominantly centred in the submucosa but with extension of abnormal blood vessels through the muscularis propria of the bowel wall.

Microscopy of the lymph nodes showed partial replacement of three nodes by similar mixed capillary and cavernous haemangioma with plump endothelial cells lining the vascular spaces but no evidence of malignancy (fig 3 ).

\section{Discussion}

Vascular tumours of the gastrointestinal tract are rare but well documented. This case is 


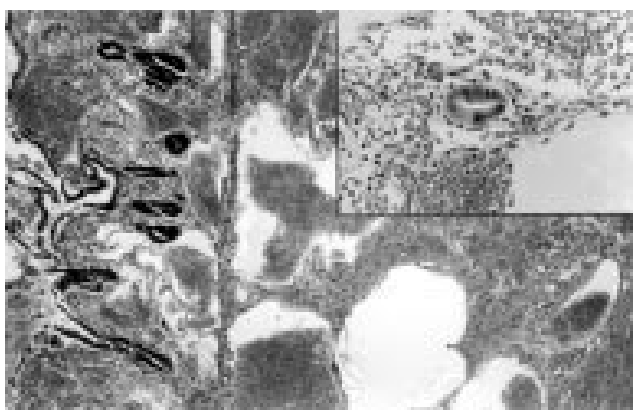

Figure 2 fejunal haemangioma. Low power view showing large (cavernous) spaces partly filled with red blood cells in the submucosa and mucosa (haematoxylin and eosin stained; magnification, $\times 16)$. The inset shows the disrupted mucosa occupied by endothelial-lined vascular spaces surrounding a single small crypt (haematoxylin and eosin stained; magnification, $\times 100)$.

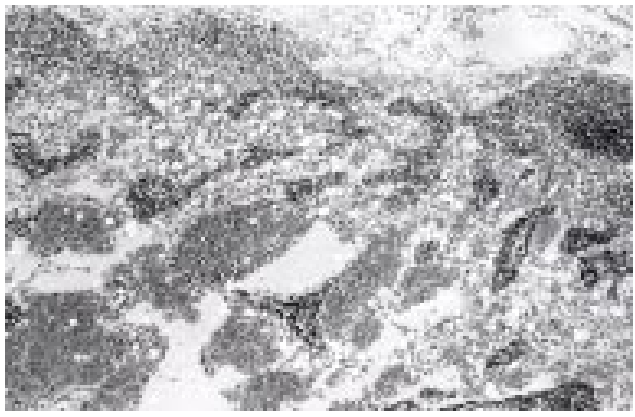

Figure 3 Vascular lesion in mesenteric lymph node. The node is almost completely replaced by large, blood filled spaces that separate thin trabeculae of lymphoid tissue and a rim of atrophied cortex (haematoxylin and eosin stained; magnification, $\times 40$ ).

unusual if not unique because of the coexistent involvement of local lymph nodes. In general, benign vascular tumours of lymph nodes are very rare; however, various forms of so called vascular transformation have been described. Solitary focal nodal haemangiomatoids are lesions that have been reported in axillary lymph nodes from patients with breast cancer. ${ }^{9}$ Nodular spindle cell vascular transformation has been reported in retroperitoneal lymph nodes from patients with renal cell carcinoma. ${ }^{10}$ Pan nodal vasodilation is said to have a particular association with ulcerative colitis. ${ }^{9}$ The pathogenesis of these conditions is obscure and none of these predisposing entities is implicated in this case. Vascular transformation of lymph nodes has also been reported in association with venous obstruction and congestion, ${ }^{11}$ but once again there was nothing to suggest that this was the cause here.

Although multiple systemic haemangiomas have been reported in entities such as blue rubber bleb syndrome, ${ }^{4}$ which is a hereditary cutaneous syndrome characterised by cavernous haemangiomas of skin and gastrointestinal tract that are thought to represent hamartomas, to our knowledge this is the first reported case of coexistent solitary small bowel and local lymph node haemangiomas. In the absence of any other specific cause we believe the most plausible explanation is hamartomatous overgrowth of blood vessels in the intestine and lymph nodes. The combination of a small bowel neoplasm and enlarged lymph nodes could have led to a mistaken diagnosis of malignancy at laparatomy and to inappropriately radical surgery.

We are grateful to Mrs J Dixon and Mrs A Ray for preparing the manuscript.

1 Boyle L, Lack EE. Solitary cavernous hemangioma of small intestine. Case report and literature review. Arch Pathol Med Lab 1993;117:939-41

2 River L, Silverstein J, Tope JW. Benign neoplasms of the small intestine. A critical comprehensive review of 20 new cases. International Abstracts of Surgery 1956;102:1-38.

3 Mako EK. Small-bowel hemangiomatosis in a patient with Maffucci's and blue-rubber-bleb-nevus syndromes. $A m \mathcal{F}$ Roentgenol 1996;166:1499-500.

4 Golitz LE. Heritable cutaneous disorders which affect the gastrointestinal tract. Med Clin North Am 1980;64:829-46.

5 Weinstein EC, Moertel GC, Waugh JM. Intussuscepting haemangiomas of the gastrointestinal tract. Ann Surg 1963; 157:265-70.

6 Murray-Lyon IM, Doyle D, Philpott RM, et al. Haemangiomatosis of the small and large bowel with histological malignant change. F Pathol 1971;105:295-7.

7 Jackson AE, Jr, Peterson C, Jr. Hemangioma of the small intestine causing protein-losing enteropathy. Ann Intern Med 1967;66:1190-6.

8 Bandler M. Haemangiomas of the small intestine associated with mucocutaneous pigmentation. Gastroenterology 1960; 38:641-5.

9 Lott MF, Davies JD. Lymph node hypervascularity: haemangiomatoid lesions and pan-nodal vasodilation. $\mathcal{f}$ Pathol 1983;140:209-19.

10 Cook PD, Czerniak B, Chan JKC, et al. Nodular spindle-cell vascular transformation of lymph nodes. A benign process occurring predominantly in retroperitoneal lymph nodes draining carcinomas that can simulate Kaposi's sarcoma or metastatic tumour. Am f Surg Pathol 1995;19:1010-20.

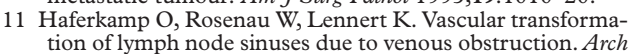
Pathol 1971;92:81-3. 